\title{
e-Migrinter
}

$20 \mid 2020$

Hospitalité et migration

\section{Quand les militants font le procès du « délit de solidarité »}

\section{Kelly Loiseau}

\section{(2) OpenEdition}

Journals

Édition électronique

URL : https://journals.openedition.org/e-migrinter/2337

DOI : 10.4000/e-migrinter.2337

ISSN : 1961-9685

Éditeur

UMR 7301 - Migrinter

Référence électronique

Kelly Loiseau, "Quand les militants font le procès du « délit de solidarité » », e-Migrinter [En ligne], 20 | 2020, mis en ligne le 23 juillet 2020, consulté le 20 mai 2021. URL : http://journals.openedition.org/emigrinter/2337 ; DOI : https://doi.org/10.4000/e-migrinter.2337

Ce document a été généré automatiquement le 20 mai 2021.

Tous droits réservés 


\title{
Quand les militants font le procès du « délit de solidarité »
}

\author{
Kelly Loiseau
}

\section{Introduction}

1 Depuis quelques années, dans le cadre de la politique migratoire en France, l'attention s'est portée de manière vive sur ce qu'il est d'usage d'appeler le " délit de solidarité » et, ce dans toutes les sphères de la société. Les poursuites et condamnations de citoyens accordant une aide désintéressée à des migrants, quelle que soit la régularité de leur situation, ont fait l'objet d'un traitement médiatique intense. En témoignent, les figures de Cédric Herrou, de Pierre-Alain Mannoni et des « 7 de Briançon » qui agissent comme autant d'illustrations des formes que peuvent prendre la solidarité envers les migrants sans papiers et sa répression. Néanmoins, l'attention ne s'est pas focalisée uniquement sur la médiatisation des «aidants" à la frontière franco-italienne. Les instances juridiques ont également fait l'objet de cette couverture. En cela, l'année 2018 marque un nouveau tournant dans la définition juridique dudit délit. Le Conseil Constitutionnel reconnaît, en effet, le principe de fraternité et propose ainsi une révision partielle de l'article de loi suite à la question prioritaire de constitutionnalité (QPC) qui lui a été posée. Ainsi, comme nous le verrons par la suite, c'est au sein de l'arène judiciaire que se donnent à voir les rapports de force en jeu entre les citoyens et associations qui appellent de leur vœu la suppression du délit et l'impératif de contrôle des frontières et de l'immigration illégale porté par l'État. Tout d'abord, il est utile de saisir l'origine militante de l'expression "délit de solidarité » et ce qu'elle désigne, avant de se pencher sur sa résistance aux multiples réformes censées faire abroger le délit. Ensuite, à travers le cas de la $\mathrm{QPC}$, nous verrons comment la société civile a recours au droit pour dénoncer les défauts d'une loi potentiellement inconstitutionnelle. Et nous terminerons par une analyse des changements et des permanences présents dans la nouvelle disposition. 


\section{Présentation et évolution dudit délit}

\section{Le « délit de solidarité » : un slogan politique}

2 Le « délit de solidarité » est une expression déroutante autant du fait de l'association paradoxale du mot délit avec celui de solidarité que par son utilisation tous azimuts. Les médias, les associations, les institutions de défense des droits mais également la sphère politique que ce soit pour nier son existence ou affirmer son abolition, l'emploient constamment. Pour autant, le délit de solidarité, présenté comme tel, ne possède aucune existence juridique si ce n'est de renvoyer au délit d'aide à l'entrée, au séjour et à la circulation des étrangers irréguliers défini dans l'article L.622-1 du Code d'entrée et de séjour des étrangers et du droit d'asile (CESEDA) :

« Toute personne qui aura, par aide directe ou indirecte, facilité ou tenté de faciliter

l'entrée, la circulation ou le séjour irréguliers, d'un étranger en France sera punie

d'un emprisonnement de cinq ans et d'une amende de 30000 Euros. ».

Alors, d'où vient cette expression? Elle a été utilisée pour la première fois par le Groupe d'Information et de Soutien aux Immigrés (GISTI) dans le milieu des années $1990^{1}$. Cette période était particulièrement marquée par la multiplication du nombre d'interpellations des «aidants " ainsi que par la forte mobilisation d'étrangers sans statut qui revendiquaient leur régularisation. L'été 1996 en garde de profonds stigmates notamment du fait de l'occupation suivie de l'expulsion musclée des « sans-papiers » de l'Église Saint Bernard dans le XVIIIe arrondissement de Paris. En ce sens, l'intention première est de dénoncer ces poursuites conduites à l'encontre de particuliers et d'associations qui apportent de manière désintéressée de l'aide à des étrangers en situation irrégulière. Autrement dit, ce néologisme pointe du doigt l'existence dans le droit français d'un amalgame entre « aidants » et «passeurs » qui en ferait un business. Par ailleurs, l'expression a profité d'un pic de popularité en 2009 avec la sortie du film Welcome de Philippe Lioret. L'histoire d'un maître-nageur de Calais qui aide en secret un jeune réfugié kurde a su susciter les réactions du ministre de l'Immigration, de l'Intégration et de l'Identité nationale de l'époque, Éric Besson².

\section{Un délit qui résiste au temps et aux réformes}

3 En 2019, les dénonciations des associations et des institutions de défense des droits perdurent et ce malgré les multiples modifications des articles du CESEDA et la suppression annoncée du délit de solidarité en $2012^{3}$. D'autant plus que la condamnation de l'aide à l'entrée, à la circulation et au séjour irrégulier s'inscrit dans un temps long. Serge Slama le désigne d'ailleurs comme "un délit d'une autre époque » vu qu'il voit le jour dans le décret-loi du 2 mai 1938, autant dire dans un contexte particulièrement xénophobe, avant d'être repris tel quel à la Libération et d'être intégré dans le CESEDA en 2004. Les modifications apportées au fil des lois sont essentiellement de trois ordres. Les réformes du Code se sont, tout d'abord, accompagnées d'un alourdissement des peines. Les engagements européens et internationaux qui ont suivi, ont conduit à l'extension du champ d'application géographique du délit. Ils étendent, aujourd'hui, le régime des sanctions à l'ensemble des pays de l'espace Schengen ainsi qu'aux États signataires de la Convention de Palerme contre le trafic d'êtres humains. Enfin, une grande partie des modifications porte sur l'élargissement des exemptions pénales qui définissent les cas d'exception 
pour lesquels l'aidant n'est pas poursuivi. C'est dans cette optique qu'a été réédité, le 31 décembre 2012, l'article L.622-4 qui endosse la lourde ambition de sonner l'abrogation du délit de solidarité selon les propos de Manuel Valls. En plus des immunités familiales qui permettent de protéger les membres de la famille proche, le troisième et nouvel alinéa, énumère les autres situations pour lesquelles le délit n'est pas avéré. De ce fait, n'est pas poursuivie :

«toute personne physique ou morale, lorsque l'acte reproché n'a donné lieu à aucune contrepartie directe ou indirecte et consistait à fournir des conseils juridiques ou des prestations de restauration, d'hébergement ou de soins médicaux destinées à assurer des conditions de vie dignes et décentes à l'étranger, ou bien toute autre aide visant à préserver la dignité ou l'intégrité physique de celui-ci. ».

À savoir que les exemptions pénales listées précédemment ne s'appliquent que pour l'aide au séjour et ne prennent en compte ni l'aide à la circulation ni celle à l'entrée.

4 À l'évidence, le délit de solidarité a su résister au temps et aux changements au vu des affaires $^{4}$ qui ont été relayées par les médias: en décembre 2015, une femme accompagnant deux ressortissants érythréens en gare d'Antibes a été condamnée à 1500 euros d'amende; le mois suivant, un Britannique qui conduisait une fille de 4 ans vivant dans le bidonville de Calais auprès de sa famille en Grande-Bretagne a été inquiété pour "délit d'aide au séjour et à la circulation" avant d'être finalement condamné pour «mise en danger de la vie d'autrui » car elle ne portait pas de ceinture de sécurité. La disposition de l'article L.622-4 ne permet manifestement pas de différencier les particuliers qui apportent une aide dans un but purement humanitaire ou par solidarité, des organisations lucratives qui sont pourtant les cibles de la répression. En somme, le projet de loi de 2012 n'a pas atteint son objectif affiché.

\section{Le droit français crée des « délinquants solidaires »}

\section{Retourner le droit contre l'État}

La dernière réforme du Code sur le délit d'aide, en date du 11 septembre 2018, s'inscrit dans une toute autre démarche. Elle fait suite à la décision rendue par le Conseil Constitutionnel le 6 juillet de la même année pour une QPC qui lui a été posée. La nature même d'une QPC qui

« est le droit reconnu à toute personne qui est partie à un procès ou une instance de soutenir qu'une disposition législative porte atteinte aux droits et libertés que la Constitution garantit ${ }^{5}$ »

est profondément contestataire. Ici, Cédric Herrou et Pierre-Alain Mannoni, tous deux condamnés respectivement à 4 mois et 2 mois d'emprisonnement par la Cour d'Appel d'Aix-en-Provence soutiennent que deux articles (art. L.622-1 et L.662-4) du CESEDA vont à l'encontre du principe de fraternité pourtant inscrit dans la Constitution.

Pour restituer les faits dans les grandes lignes, Cédric Herrou, agriculteur de la Roya, est condamné, dès la première instance, pour avoir

«facilité l'entrée, la circulation et le séjour irréguliers de plusieurs étrangers se trouvant dépourvus de titre de séjour (environ 200) sur le territoire national » (TGI de Nice, 10 février 2017).

Son engagement auprès des migrants fait, par ailleurs, l'objet d'un film documentaire qui a été porté sur grand écran 6 . Pierre-Alain Mannoni, enseignant-chercheur, est, quant à lui, rendu coupable d'avoir transporté et hébergé trois Érythréennes. Bien que 
le caractère désintéressé et solidaire soit attesté par la juridiction de première instance, la Cour d'Appel d'Aix-en-Provence ne leur accorde pas l'immunité du fait de leur militantisme. Le raisonnement de la Cour associe délibérément leurs actions solidaires et militantes à une entrave «visant à faire échec à la politique migratoire menée par l'État ». Face aux condamnations dont ils font l'objet, les deux requérants utilisent le droit comme bouclier (Israël, 2018). Dès lors, la justice devient un levier d'action dans les mains de la société civile pour contester une loi jugée illégitime et qui plus est possiblement inconstitutionnelle. Par ailleurs, leur entreprise s'inscrit dans une action collective plus étendue : des associations de défense des droits de l'Homme, le collectif des Délinquants Solidaires ainsi que la Commission nationale consultative des droits de l'Homme (CNCDH) apportent leurs propres observations pour étayer l'affaire.

\section{Entre l'immunité et la condamnation d'un aidant, il n'y a qu'un pas}

7 Ces soutiens se dressent à l'unisson contre la rédaction quelque peu obscure et restrictive des dispositions de l'article L.622-4 du CESEDA. Sont pointées du doigt les exemptions pénales prévues. Ne s'appliquant que pour la seule aide au séjour, elles ne protègent ni l'aide à la circulation ni à l'entrée quand bien même elles aient été conduites dans un but purement humanitaire et solidaire. Dit autrement, conduire un migrant d'un point à un autre à l'intérieur du territoire national y compris vers une Préfecture pour qu'il puisse y déposer sa demande d'asile ou bien indiquer la route la moins dangereuse à ceux qui tentent une traversée périlleuse à travers les Alpes sont autant de situations d'aide qui peuvent être poursuivies voire condamnées. D'autre part, concernant l'aide au séjour, deux conditions doivent être réunies pour éviter une condamnation. Premièrement, ne pas avoir reçu de contrepartie. Il conviendrait d'ajouter que la loi laisse la porte ouverte à de multiples interprétations quant à la nature de ces contreparties. Que l'on songe un instant à un militant du Réseau Education Sans Frontières (RESF) qui a été accusé d'aide au séjour irrégulier car la famille qu'il hébergeait, participait aux tâches ménagères ${ }^{7}$. Deuxièmement, l'aide apportée doit s'inscrire dans la liste des actes tels que des « conseils juridiques ou des prestations de restauration, d'hébergement ou de soins médicaux » ou "toute autre aide visant à préserver la dignité ou l'intégrité physique ». Et c'est là que s'immisce une nouvelle faille. La notion de dignité et ses contours flous exposent les inquiétés à l'arbitrage des juges. En cela, les dispositions n'offrent pas une protection suffisante pour couvrir toutes les formes que peut revêtir l'assistance apportée de manière désintéressée. Qu'en est-il des cours de français ou des recharges de téléphone ? Cellesci ne rentrent pas forcément dans la typologie des actes jugés nécessaires à la préservation de la dignité des personnes. En deux mots, ce sont les caractéristiques restrictive et imprécise de l'article qui sont visées. En plus des défauts soulevés sur les exemptions (art. L.622-4), la plaidoirie des associations attire tout autant l'attention sur les dangers de la définition même du délit d'aide dans le Code. L'article L. 622-1 considère toute aide comme un délit jusqu'à preuve du contraire. Autrement dit, l'inquiété doit apporter la preuve qu'il entre dans l'une des cases des exemptions précitées. $\mathrm{La}$ CNCDH alerte sur les risques « d'autocensure de la part des citoyens et des bénévoles associatifs» (CNCDH, 2017 : 9). 


\section{La justice peut-elle faire bouger les lignes?}

\section{La position du Conseil Constitutionnel}

8 Dans la décision du Conseil Constitutionnel qui était attendue avec impatience par les requérants ainsi que par l'ensemble des acteurs associatifs et institutionnels, il est reconnu le principe de fraternité inscrit dans la devise nationale comme valeur constitutionnelle, et en se fondant sur celui-ci, le Conseil étend également les immunités à l'aide à la circulation. Dit autrement, cette nouvelle protection constitutionnelle donne

«la liberté d'aider autrui dans un but humanitaire, sans considération de la régularité de son séjour sur le territoire national » (Décision n 2018-717/718 QPC du 6 juillet 2018).

Néanmoins, il est aussi rappelé que la sauvegarde de l'ordre public est également un objectif à valeur constitutionnelle et qu'elle passe par la lutte contre l'immigration illégale. Le Législateur se doit, donc, d'assurer un équilibre entre le principe de fraternité et la sauvegarde de l'ordre public. De cette manière, le Conseil rappelle la souveraineté de l'État et son droit de contrôler l'accès au territoire. Concernant l'élargissement de l'immunité à l'aide à la circulation, la décision s'appuie sur des précédentes affaires dans lesquelles le transport est défini comme un accessoire à l'aide au séjour. Le cas de Mannoni en est, d'ailleurs, un exemple. Le juge du tribunal de grande instance (TGI) de Nice a décrété que

«[...] force est de constater que la circulation des trois migrantes mise en œuvre par le prévenu n'était que le préalable indispensable à l'aide à leur séjour, couvert par l'immunité prévue à l'article L. 622-4 [...] ».

De ce fait, conduire un étranger en situation irrégulière vers le lieu de sa mise à l'abri ne fera plus l'objet de condamnations. Cependant, en dépit des arguments avancés sur les imprécisions de l'alinéa 3, les Sages ${ }^{8}$ émettent une réserve d'interprétation sur cette disposition. À leurs yeux, la rédaction est suffisamment claire pour être interprétée comme s'appliquant à "tout autre acte d'aide apportée dans un but humanitaire ». Toutefois, la QPC marque une réelle étape en reconnaissant l'importance du principe de fraternité. Certes, un écart persiste entre la position du Conseil constitutionnel et les revendications des militants et des institutions de défense des droits mais, il y a un progrès.

\section{Entre changements effectifs et immobilité : ce que dit la nouvelle rédaction du Code}

9 Sur les pages du Code, la transposition de la décision du Conseil Constitutionnel par le Législateur est la suivante :

« ne peut donner lieu à des poursuites pénales sur le fondement des articles L. 622-1

à L. 622-3 l'aide à la circulation ou au séjour irréguliers d'un étranger lorsqu'elle est le fait: $3^{\circ}$ De toute personne physique ou morale lorsque l'acte reproché n'a donné lieu à aucune contrepartie directe ou indirecte et a consisté à fournir des conseils ou accompagnements juridiques, linguistiques ou sociaux, ou toute autre aide apportée dans un but exclusivement humanitaire $^{9}[. .]$.$» .$

Entrée en vigueur le 11 septembre 2018, la lecture de la nouvelle disposition suggère que le Législateur a bien pris en compte la décision portée par le Conseil et est même 
allé au-delà de celle-ci. Avec des termes plus généraux, il en modifie le contenu de manière à rendre la liste des actes non réprimés plus large en gommant notamment la notion de dignité et d'intégrité physique. Cependant d'un autre côté, il prescrit que l'immunité sera accordée à «toute autre aide apportée dans un but exclusivement humanitaire» et cette mention d'exclusivité semble faire écho au raisonnement emprunté par la Cour d'appel d'Aix-en-Provence, à savoir condamner les actions militantes. Annalisa Lendaro, qui étudie l'expression de la solidarité envers les migrants dans la vallée de la Roya comme une forme de désobéissance civile, y voit :

« [un] procès politique exemplaire [qui] sanctionne le militantisme entendu comme

l'ensemble des formes de solidarité qui visent, en même temps qu'à prêter secours à

des personnes en détresse, à souligner les défaillances et les abus de pouvoir de

l'État » (Lendaro, $2018: 181$ ).

De la même manière, le Défenseur des droits s'inquiète de futures condamnations qui suivraient cette dérive au point d'aboutir à l'émergence d'un délit d'opinion ${ }^{10}$.

Il est d'autant plus intéressant de se pencher sur les dispositions qui ont résisté au temps et aux réformes depuis leur version initiale. Et c'est précisément sur ce fil que se noue le cœur du débat autour du délit de solidarité. En effet, tous les efforts des acteurs se concentrent sur la définition des exemptions pénales que ce soit pour dénoncer leur protection insuffisante, les élargir ou bien les censurer. Or, en creux, cela conduit inévitablement à s'interroger sur ce qui doit être et rester pénalisable. Prenons l'expression " contrepartie directe ou indirecte » qui, bien que vivement critiquée de toute part, est restée inchangée depuis sa première inscription dans le CESEDA en $2005^{11}$. Il est légitime de se demander pour quelles raisons la législation française a constamment refusé d'introduire la référence au but lucratif qui apparaît pourtant être l'élément matériel qui permet de différencier une aide désintéressée d'une filière de passeurs. Or, comme le rappelle le Défenseur des droits et la $\mathrm{CNCDH}$, cette référence est présente dans le droit européen et international: la Directive européenne du 28 novembre 2002 incrimine uniquement l'aide apportée «sciemment » et « dans un but lucratif ». En cela, le droit français ne se plie pas à ses engagements internationaux. Ensuite, est-ce que le souvenir des condamnations de la France pour esclavage moderne, en 2005 et 2012, expliquerait l'incapacité de dépasser la notion évasive de contrepartie? En effet, à deux reprises, la France a été condamnée par la Cour Européenne des Droits de l'Homme (CEDH). Son cadre législatif était jugé inefficace dans la lutte contre l'esclavage domestique. Dans les deux affaires concernées ${ }^{12}$, des jeunes filles mineures originaires du Togo et du Burundi ont été réduites à un état de servitude dans les foyers où elles dormaient. L'idée sous-jacente serait d'éviter l'exploitation de la vulnérabilité des étrangers en situation irrégulière. Dans cette optique, les associations proposent plutôt de poursuivre les actes où la contrepartie est disproportionnée à l'aide accordée afin d'y inclure les formes de participations des « aidés ».

11 Quant à l'aide à l'entrée sur le territoire national, la logique sécuritaire adoptée par l'État ne permet guère d'envisager sa possible inscription dans le champ des exemptions pénales. À ce sujet, le Défenseur des droits tient à rappeler que l'entrée de certaines catégories de migrants ne peut être considérée comme irrégulière : c'est le cas notamment des mineurs. Ainsi :

« condamner l'aide au séjour irrégulier d'un mineur n'a pas de sens dès lors que les mineurs ne sont pas tenus par l'obligation de détenir un titre de séjour et ne peuvent donc être en situation de séjour irrégulier sur le territoire » (Défenseur des Droits, $2018: 67)$. 
Dans la même logique, les étrangers relevant de la Convention de Genève ne peuvent faire l'objet de sanctions pénales alors pourquoi ceux qui les aident peuvent-ils être inquiétés?

\section{Conclusion}

12 La décision du 6 juillet 2018, qui consacre notamment le principe de fraternité, est une énième illustration des rapports de force entre les autorités publiques et les militants. Tandis que les premières campent sur leurs positions (négation du délit et annonce de sa suppression) les seconds souhaitent la conformité du droit français aux engagements européens et internationaux ou une redéfinition totale du délit d'aide. Bien que la nouvelle rédaction du Code ne vienne pas à bout du délit de solidarité, la QPC montre dans quelle mesure la justice peut être une alliée de la société civile pour faire bouger les lignes. Cependant, l'impératif de contrôle des flux migratoires mené par l'État limite indéniablement la portée de l'immunité humanitaire. Il semblerait, d'ailleurs, que ce n'est plus qu'à travers ce contrôle que l'État a l'impression de faire respecter sa souveraineté. Enfin, il convient de préciser que la répression de la solidarité envers les étrangers revêt d'autres formes. Les aidants bénévoles ou associatifs peuvent être poursuivis pour mise en danger d'autrui, diffamation, outrage, injure ou bien infraction au code de l'urbanisme pour avoir construit un abri de fortune. Leurs actions peuvent, également, être entravées par des contraventions routières régulières ou bien par la fermeture de locaux pour raisons sanitaires. L'utilisation de ces outils juridiques qui n'entrent pourtant pas dans le cadre du droit des étrangers, montre de quelle manière les pouvoirs publics tentent d'empêcher l'expression de la solidarité. Le sociologue Vincent Geisser mettait déjà en avant en 2009 ces pratiques répressives qui, selon lui, se situent "à la frontière de la légalité » (Geisser, 2009: 13). Dans un contexte de forte mobilisation militante contre le délit de solidarité, l'auteur pose à plat une interrogation qui garde toute sa pertinence encore aujourd'hui. Il se demande si le combat se situe réellement dans l'arène judiciaire. Autrement dit, est-ce que le délit de solidarité disparaîtrait complètement si les articles de loi du CESEDA arrivaient à différencier sans faille l'aide désintéressée des réseaux de passeurs sachant qu'une politique répressive globale est conduite?

\section{BIBLIOGRAPHIE}

CNCDH (2018) Délit de solidarité ou devoir de fraternité ? Avis sur La situation des personnes migrantes à la frontière franco-italienne, 19 juin 2018, pp. 28-32.

CNCDH (2017) Avis sur Mettre fin au délit de solidarité, 18 mai 2017, 12 p.

Décision n²018-717/718 QPC du 6 juillet 2018

Défenseur des droits (2018) Une pénalisation protéiforme de l'aide aux exilés, Rapport sur Exilés et droits fondamentaux, trois ans après le rapport Calais, décembre 2018, pp. 65-69. 
Geisser, Vincent (2009) Délinquance humanitaire : Du "délit de solidarité" au "devoir de délation", Migrations Société, n 123-124, pp. 7-18.

Israël, Liora (2009) L'arme du droit, Paris, Presses de Sciences Po, 133 p. (Coll. Contester).

Lendaro, Annalisa (2018) Désobéir en faveur des migrants, Répertoires d'actions à la frontière franco-italienne, Journal des Anthropologues, n 152-153, pp. 171-192.

Lochak, Danièle (2017). La solidarité, un délit ?, Revue Projet, n 358, pp. 56-62.

Mathonnet, Paul (2018) Le délit de solidarité à l'épreuve du principe de fraternité, Plein droit, $\mathrm{n}^{\circ} 118$, pp. 41-44.

Slama, Serge (2017) Délit de solidarité : actualité d'un délit d'une autre époque, Lexbase Hebdo, $\mathrm{n}^{\circ} 456,14$ p. (Edition publique).

\section{NOTES}

1. Le GISTI met en permanence à jour un dossier consacré au délit de solidarité sur son site internet. Dans leurs archives, l'utilisation la plus ancienne de l'expression date du 12 mars 1998 dans un communiqué intitulé "Après le délit d'hospitalité (Debré) le délit de solidarité (Chevènement) ». Entre autres, dans l'article « Délit de solidarité : les origines » (2009) issu de la revue Plein droit, le GISTI est à l'origine d'un premier «Manifeste des délinquants de la solidarité » qui date de 1995.

2. Bénabent, Juliette (2009) “Pas “Welcome”, Eric Besson...” in Télérama [en ligne, consulté le 23 mars 2019] URL : https://www.telerama.fr/cinema/welcome-eric-besson-en-croisade,40447.php 3. Communiqué de presse de Manuel Valls du 12 décembre 2012 «Adoption du projet loi relatif à la retenue pour vérification du droit au séjour et à l'abrogation du délit de solidarité ».

4. Le Gisti tient à jour un dossier en ligne sur le délit de solidarité dans lequel sont recensés les condamnations des « aidants » solidaires.

5. Définition issue de la page web du Conseil Constitutionnel (https://www.conseilconstitutionnel.fr/decisions/la-qpc).

6. Film documentaire « Libre » réalisé par Michel Toesca et sorti en septembre 2018.

7. Hilel Roger (16 juillet 2015) «Denis Lambert : un " juste " solidaire des sans-papiers", l'Humanité consulté sur https://www.humanite.fr/denis-lambert-un-juste-solidaire-des-sanspapiers-579512

8. Les neufs membres du Conseil Constitutionnel sont appelés des « Sages ».

9. C'est nous qui soulignons.

10. Le 16 janvier 2019, un homme est condamné à deux mois de prison avec sursis par la Cour d'appel d'Aix-en-Provence au motif que son acte s'inscrit dans « une démarche d'action militante ».

11. Ordonnance 2004-1248 2004-11-24 JORF 25 novembre 2004 entrée en vigueur le $1^{\text {er }}$ mars 2005.

12. CEDH, Siliadin c. France, 26 juillet 2005 et CEDH, C.N.\&V. c. France, 11 octobre 2012. 


\section{RÉSUMÉS}

L'année 2018 marque un tournant dans la définition juridique du « délit de solidarité » avec la reconnaissance du principe de fraternité par le Conseil Constitutionnel. À travers l'étude de cette décision, cet article propose une illustration des rapports de forces qui se jouent dans l'arène judiciaire entre d'un côté, les citoyens et associations qui exigent la suppression de ce délit et, de l'autre, l'État guidé par un impératif : celui du contrôle des frontières et de l'immigration illégale.

\section{INDEX}

Index géographique : France, Union européenne

Mots-clés : délit, solidarité, droit de l'immigration, justice

\section{AUTEUR}

\section{KELLY LOISEAU}

Master I - Migrations Internationales / Université de Poitiers

kelly.loiseau@gmail.com 